dem Ausdruck "Blutschande" stigmatisiert werden darf 1.: Uebrigens ist noch der Ausdruck אלמנה Vers 11 zu beachten, den Juda verwendet; nach dem altassyrischen Recht wäre Tamar als almattu berechtigt gewesen, zu gehen, wohin sie wollte, während Juda sie zu ihrem Vater sendet.

\section{Zur Akkadischen Weisheitsliteratur.}

Von Arthur Ungnad.

In Bd. I 2 der Publications of the Babylonian Section des University Museum zu Pbiladelphia (abg. UM) veröffentlicht H. F. Lutz unter Nr. 116 einen bilinguen neubabylonischen Text (CBM 4507), der zur Beschwörung böser Geister diente. Nach den Schlussworten

Ein böser alû, der sich auf dem Menschen lagert und wie ein Esel lostrabt ${ }^{2}$, bist du;

"Ein böser alû, der Opferspende nicht kennt und Mehlspende ${ }^{3}$ nicht hat, bist du ${ }^{4}$

heisst es, gewissermassen als Unterschrift:

(54) e-riš ti ki i ši e-pir ì ${ }^{4}$ ku-ub-bit ina an-ni-im-ma ilum ha-di-iš ta-a-bi eli ilušamaš i-rab-šu dum-ḳu

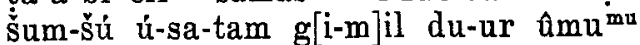
amta ina bîti e tu-kab-bit.

Damit endet die Tafel. Dieser letzte $A b-$ schnitt, der mit der Beschwörung gar nichts zu tun hat, erweist sich als ein Duplikat zu den von MaCMILLAN in BA V, S. 558 behandelten Texten ${ }^{5} \mathrm{~K} 7897$ (Rs., eigentlich Vs. $10 \mathrm{ff}$.) und 33851 II $11 \mathrm{ff}$. und ergänzt jene in einigen Zeilen. Zwischen e-riš und e-pir bietet K 7897 kit-tu und 33851 lit-tú, wofür hier das mir un. klare ti ki $i$ ši steht. Ebenso unklar ist das in Z. 57 stehende šum-šu statt šub-šu in 33851. Setzen wir die besseren Lesungen der Londoner Texte ein, so ist zu übersetzen:

(54) Suche die Wahrheit, versorge und ehre ${ }^{6}$, über dieses freut sich der Gott (darüber), angenehm ist es Šamaš, er gibt dir dafür Gutes.

1 Benzinger Arch. S. 288 entnimmt Gen. 38 richtig, $\mathrm{daB}$ dort eine alte Sitte vorausgesetzt ist, in welcher die Verpflichtung (die kinderlose Witwe zu heiraten) auf den Schwiegervater ausgedehnt ist. Nur ist jetzt die alte Sitte" als ein Rechtsinstitut aufzufassen, das dem altassyrischen parallel war.

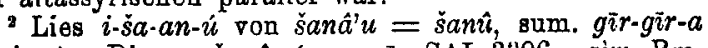
(giggir-a). Dieses šanu (sum.gīr SAI 3306; gim Rm. 2.588:31 b; im Br. 4821) ist wohl ein Verb der Bewegung (vgI. DU = gim, gir = šanû SAI 3347); es dürfte eine ânnlich Bedeutung wie lasâmu haben (vgl. SAI 3304. 3307). Der amél šân̂u "Traber" (UM $\nabla$ 145:8) ist wohl ungefähr mit dem amel lâsimu "Lüufer" identisch.

${ }^{3}$ Lies ma-as-ha-ta.

- Kopie hul.

5 Vgl. auch Zimmern, ZA 23, $367 \mathrm{ff}$.

- Objekt "deine Eltern" o. i. Vgl. die Kontraktliteratur über die Verpflegung alter Angehöriger.
Vollführe Wohltaten und sei gefällig immerdar,

Die Magd im Hause beschwere ${ }^{1}$ nicht! Zusatz des Herausgebers:

Ist vielleicht $\mathrm{i}$-lim zu lesen? tiki etwa $=$ Ausfluss, also "die göttliche Emanation".

Sollte nicht in šumšu ein Hinweis auf aspirierte Ausprache des šubšū vorliegen? F. E. P.

\section{Die ältesten indogermanischen Sprachreste.} Von Heinrich Hein.

Als ältestes Auftreten indogermanischer Sprachen in der Geschichte galt bisher das Vorkommen arischer Götternamen, Eigennamen und Bezeichnungen in den Texten von Boghazköi und Tell Amarna. Im folgenden soll gezeigt werden, dass ganz wesentlich früher schon der indogermanische Sprachstamm sich bemerkbar macht und zudem bei demjenigen Volk, dass der Kultur des Orients auf Jahrtausende seinen Stempel aufgedrückt hat und bis heutigestags in der Weltkultur nachwirkt, - den Sumerern.

Des beschränkten Raumes halber soll hier nur summarisch der Lautbestand des Sumerischen betrachtet und anschliessend eine beschränkte-Auswahl yon Wurzelvergleichungen gegeben werden.

Allgemein anerkannt wird wohl, dass das Snmerische stark abgeschliffen ist, $d$. $h$. dass es die Endungen bei Hauptwort, Adjektip und Verbum zum grossen Teil verloren hat und so vielfach den nackten Stamm verwendet. Auch eine Abschleifung des Stammes durch Abfall der Endkonsonanten ist angenommen worden (z. B. von Delitzseh), doch führt die Sprachvergleichung zu anderem Ergebnis. Der Ausdruck zeigt zuweilen fast stenographische Kürze.

Anerkannt dürfte ferner sein, dass die Media $(b, g, d)$ sehr oft im Lautwert der Tenuis $(p, k, t)$ nahe kommt. Beweis aus Emesaltexten und auch sonst. (gašan, kašan; $d u b$, tub u. a. m.). Beweis auch dadurch, dass die vielen Lehnwörter im Assyrischen statt sumerischer Mledia oft Tenuis zeigen: balag pilakku; 2bar paršigu neben barsig; sa.bár; 4 gir kĩru; dur turrum usw.

Bei Sprachvergleichung dürfte demnach sumerisches $\bar{b}, g, d$ sowohl griech.(-lat.) $\beta, \gamma, \delta$ als auch $\pi, x, \tau$ entsprechen, und zwar im Anlaut, aber auch im Inlaut und Auslaut. Sumerisches $p, k, t$ würde nur griech.(-lat.) $\pi, x, \tau$ gleichstehen. Diese Erscheinung erinnert an die germanische Lautverschiebung, wo sich ähnlich die Media in die Tenuis wandelt, die Tenuis

"Doch wohl kaum „ehre"; auffällig ist dann der Bedentungswechsel desselben Verbs in Z. 54 und Z 58 . 
aber weiter in die Aspirata. Letzteres ist im Sumerischen nicht nachweisbar, da die Aspiraten $f$ und $y$ fehlen. $h$ ist bekanntlich vorhanden. $\vartheta$ ist unter $t$ zu finden, wofür unten Beispiele. $f$ dürfte, falls im Sumerischen vorhanden gewesen, eher durch $b$ als durch $p$ wiedergegeben sein.

Bekannt ist ferner, dass $z$ und $s$ sich zuweilen vertreten: $3 z i, s i ; z i . i r, 3 s i r ; z u ́ r, z i r r u$, surru; zuh, suh.

Auch Wechsel von $z$ und $\check{s}$ kommt vor $z i, \grave{s} i$.

Sonach wird im allgemeinen $z \operatorname{dem} \zeta, s$ dem $\sigma$ gleichzusetzen sein, aber die umgekehrte Entsprechung ist nicht ausgeschlossen. Tatsächlich ist $z$ vielfach gleich $\sigma$.

Dass sumerisches $\dot{s}$ kein genau definierter Laut ist, zeigen die Lehnwörter im Semitischen deutlich: dubsar, tupšarru; sanga, šangu; ;es si. gar, sigaru. Im sumerisch-assyrischen Gìlossar verwendet der Assyrer also $s$ wo das Volk $s$ benutzte. Das deutet auf Laute wie $\sigma \chi, \sigma x, \sigma \pi$, $\sigma F$. Auch Laute wie $\sigma \tau, \sigma \tau \rho, \sigma \pi \lambda$ kämen in Frage. Für einige dieser Laute glaube ich Hinweise gefunden zu baben.

Bekannt ist ferner, dass der Vokalreichtum des Sumerischen grösser war, als sich durch die vier Vokale der Assyrer $a, e, i, u$ wiedergeben liess. Für $a$ kämen $\alpha, o, \eta$ auch $\alpha v$ und $\alpha o$ etwa in Betracht, für $e$ ìm allgemeinen Laute wie $\alpha \iota, \eta, \varepsilon, \varepsilon \iota$, für $i: \iota, \varepsilon, \varepsilon \iota, o \iota$, für $u: o$, $\omega, \alpha v$, $\boldsymbol{\alpha}, \boldsymbol{v}, \boldsymbol{o v}$. Ein abschliessendes Urteil über diese Entsprechungen ist jedoch z. Z. nicht möglich, da, wie im Griechisch-Lateinischen z. B. o und $i$ einander entsprechen, ähnliches auch beim Sumerisch-Indogermanischen vorkommen kann: is $\mu$ pos - imber - im (im.bara) und entsprechend sonst.

Auch konsonantisch wird $u$ gebraucht für die Komplexe va, ve, vi. Beweise siehe unten bei $u$.

Dass der Lautreichtum des Sumerischen nicht zur Geltung kommt, liegt bekanntlich daran, dass die Semiten bei Uebernahme der Schrift von den Sumerern natürlich nur für die in ibrer eigenen Sprache vorhandenen Laute Zeichen übernahmen. Sie werden also später in den Glossaren sich vielfach mit annähernder Wiedergabe sumerischer Laute begnügt und der mündlichen Erläuterung des Sprachlehrers das weitere überlassen baben.

Dasselbe wird der Fall gewesen sein, wenn das Sumerische Doppelkonsonanten in eirer Silbe besass. $\mathrm{Da}$ half sich der Semit entweder durch Unterschlagung des schwächeren Konsonanten oder durch Einschiebung eines dumpfen Hilfsvokals oder durch Umstellung: indisch $k s ̌ a-$ trya Krieger wiedergegeben als satar (Wegfall von $k$, Umstellung von $r$ und a). Jndra: in. dar und in.da.ra (Umstellung bzw. Hilfsvokal). Doppelglossen (da.ár, da.ra) bei sumerischen Wörtern lassen demnach auf Doppelkonsonanten schliessen.

NachfolgendeTabelle stellt zusammenfassend dar, wie sich im allgemeinen die Laute des Sumerischen, Griechischen und Sanskrit vertreten dürften. Lateinisch ist bei Abweichung in Klammern hinzugefügt.

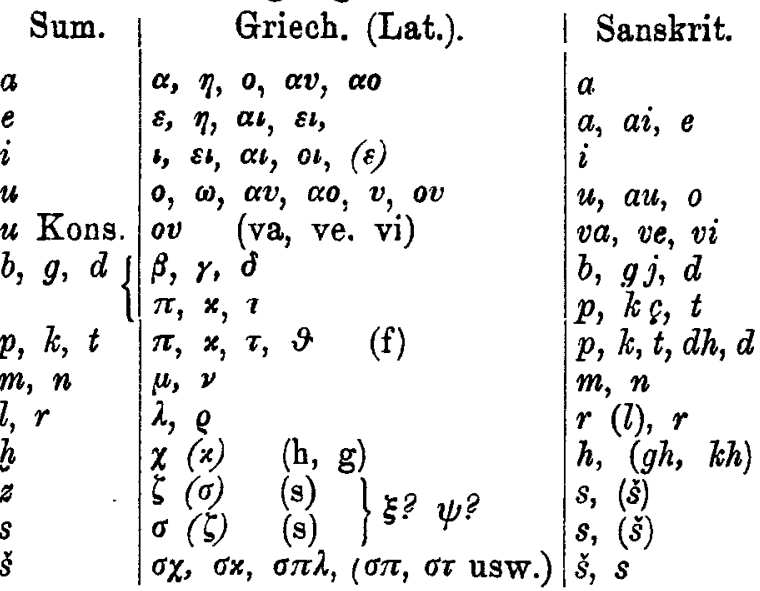

Beispiele. Die Reihenfolge und Zählung richtet sich nach dem Sumerischen Glossar von Delitzsch. Es wird nur eine kleine Auswahl charakteristischer Beispiele gegeben. "s. bedeutet sanskrit.

$\mathrm{Za}$ a: $1 a b$ Vater (als Ehrentitel) - lat. avus Grossvater, Ahn.

$3 a b$ Meer - s. $a p$ Wasser - altpreuss. ape Fluss.

apin ein Bewässerungsfahrzeug aं $\pi \dot{\eta} \nu \eta$ Lastwagen

1 ag machen; künden: - zu machen $\alpha$ $\gamma \omega$; $\mathbf{z u}$ künden $\alpha^{\prime} y y^{\varepsilon} \lambda \omega$ - lat. agere tun, treiben.

$2 \grave{a} g$ beordern; Befehl: - $\alpha^{\prime} \gamma \varepsilon \iota \nu$ anführen

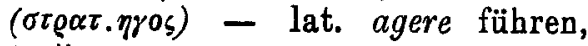
treiben.

3 àg messen: ärycı es wiegt (so und soviel) - lat. agere wägen.

Die Verwendung von $\ddot{\alpha}^{\prime} \gamma \varepsilon u$ und agere in der Bedeutung wiegen, wägen ist eine Eigentümlichkeit der Griechen and Italiker.

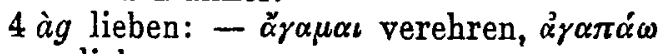
lieben

agar Flur: - s. ajra Acker - àrøós - lat. ager.

-a.da.ar Flur: - lat. ador Spelt (eine Weizenart!)

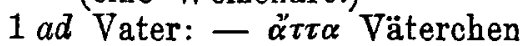

$2 a d$ ein Teil des Schiffes: - s. addi Anfang? (Bug?)

alal Wanne: - lat. alveus Wanne? 
alam Bildnis: ảhounós Anstrich, Bemalung.

1 ara zermahlen: - $\alpha^{\prime} c^{\prime} \omega$ pflügen - lat. arare pflügen.

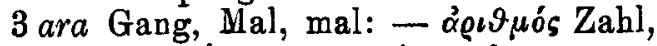

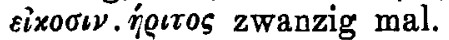

Beim Pflügen und Mahlen kommt der Mensch wohl am ehesten zum Zählen. Vgl. mahlen und -mal!

2 ara glänzend, hell: - s. aru feurig, rot? an hoch (sein), Himmel: á⿱⺌兀⿱㇒́⿱一土厶 auf, $\ddot{\alpha} \nu \omega$ droben.

anu Aehre: erinnert an $\alpha^{\prime} \nu \vartheta$ $a z u$ (auch $u z u$ ) Magier; Arzt:

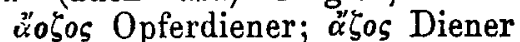

$\mathrm{Zu}$ i: ig Türflügel, Tür: - oür

igi Auge: - s. ìksh sehen.

$-i$. dè Auge: - s. veda weiss - $\varepsilon \grave{l} \delta \omega$ sehe, $i \delta \varepsilon \tilde{\nu} \nu$ sehen - lat. videre.

id Fluss: - oidźw schwellen.

$\mathrm{Zu}$ e: el hell, rein: - sil $\eta$ Sonnenlicht.

erin Zeder: - ż̊ııยós wilder Feigenbaum - s. arna Teakbaum - ornus Esche.

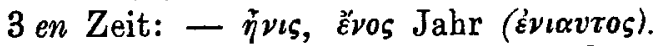

$\mathrm{Zu}$ u: ubur weibliche Brust: - lat. uber dass.

$1 u g, \dot{u} g B$ Tag, Licht: - s. oj.as Kraft, Glanz - $\alpha \dot{v} \gamma \dot{\eta}$ Glanz - A Löwe: - s. ugra mächtig, furchtbar irıins gesund - augustus.

$2 \grave{u} g$, ug mächtig: - s. ugra mächtig; furchtbar.

$3 \grave{u} g, u g$ Getier, Tier: - lat. augere mehren - lit. áugu wachsen.

$4 \grave{u} g$ Wehklage: lat. vagire wimmern u. ä.

$5 u g$ schauen (im Ablaut zu igi? ) - ỏ $\sigma \sigma \varepsilon$ aus óxjs Augen.

$6 u g($ ?) Tod, tot; morden, töten: - lat. victima Schlachtopfer, victor - got. weihan kämpfen.

7 ug uku Volk: - s. veças Haus - Foĩxos Haus - vicus Weiler.

1 ur Mensch, Diener: s. vīra Mann lat. vir.

2 ur Hund, Löwe: - keltisch ur wild, vgl. auch Auerochs? ur. $k u$, der spezielle Ausdruck für Hund, ist vielleicht $z u$ s. vrka-zend, vehrka Wolf zu stellen?

3 ur fremd, feind: - lat. vereor sich scheuen vor.

4 úr Bein; Unterkörper des Menschen, von Dingen; Wurzel - s. vāra Schweif - oṿá Schweif, Heck, őgos Steiss.

3 ur umschliessen, verscbliessen; Hürde u. ä. - s. var umschliessen - oi @os Wächter, Hüter - wwahren ".

8 ur ernten: - wंpaĩos reif, íwóaía Erntezeit.
10 ur Scham, Scheu: - s. 3 ur. (ur Fülle: - s. vära Haufen, Menge).

$2 u s ̌ A$ fliessen; fliessend machèn: - $\mathrm{s}$. vish flüssig machen, visha Saft ios (-fioós) Saft. - virus Saft. (*visos)

2 uš $A$ männl. Glied: - s. vish Exkremente.

3 uš Blut: - vgl. 2 uš and lat. vis-cera das rohe Fleisch unter der Haut.

$4 u s$ Tod, tot; Todesblick: - s. visha Gift - iós (= Firós) Gift - lat. virus Gift. *visos.

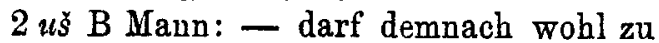
lat. vis Kraft gestellt werden.

uš Einsicht, Verstand - s. vidyá Wissen - lat. visere einsehen, Intensivform zu videre sehen.

(uš Schein: - lat. visio Erscheinung)

$1 u s ̌$ sich hart andrängen

$5 u s ̌$ dämmen (Wasser) dürften dann wohl zu s. $v \bar{a}$ weben - lat. vi.ere weben, vitis Ranke usw. in Verwandtschaft stehen, wie endlich

6 uš Liebe, Erbarmen zu: - s. vī lieben - lat. in.vitus unliebend = ungern.

$\mathrm{Zu}$ b: 1 bal A durchbrechen (Hindernis u. ä.) $\Rightarrow \pi \dot{\lambda} \lambda \lambda \omega$ rütteln, schütteln - lat. pellere?

1 bal B graben: - lat. pala Spaten.

2 bal ausgiessen: $-\beta \alpha \lambda \lambda \omega$ werfen; $\pi \alpha-$ $\lambda v ́ v \omega$ streuen - pulvis Staub.

4 bal Beil (Abkürzung von balag pilakku?) - s. piraçu Axt - $\pi \dot{\varepsilon} \lambda \varepsilon * v \varsigma$ Axt.

bal in gis az.bal Raubtierkäfig: - s. pura

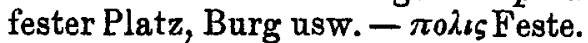

1 bur (urspr. Höhlung) Ohr, Sinn; Hohlgefäss; Tiefe, Loch; Flussbett: s. por hindurchbringen - $-\pi$ ópos Fubrt, Durchgang; Loch;insb. Strombett.

Zu p: pa Schreiber: - s. pavĩra Lanze (vgl. Griffel) - $\pi \alpha i \omega$ schlagen, stechen - lat. pavio schlagen.

( $p a$ Herr: s. pa Herr)

pa.še Reife der Feldfrucht $-\pi \varepsilon \pi \alpha i \nu \omega$ reif werden.

pala Prachtkleid u. ä. - lat. palla dass.

pel beschmutzen: - s. palvala Teich, Pfütze - $\pi \eta \lambda o_{s}$ Schlamm - lat. palus Sumpf.

Zu g: ga Haus: - a av

${ }_{g i s} g a g(?)$ Pflock: - ráyydıov Knoten. gana glänzende Wasserfliege: - yávos Glanz.

1 gal gross: (vgl. ègal =ekallu!) - s. kalya heil, gesund - xalós schön.

1 gam 1. sich neigen, beugen; 2. körperliche Zerschlagenheit: - $x_{\alpha}^{\prime} \mu \pi \tau \omega$ 


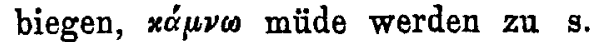
çam ermüden.

gan gebären. - s. jan zeugen, ent-

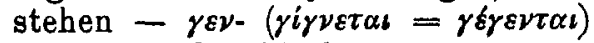
lat. genus Geschlecht.

gam gebären: - s. jami Geburt u. ä.

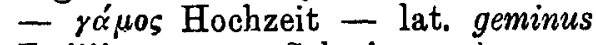
Zwilling, gener Schwiegersohn aus gemer.

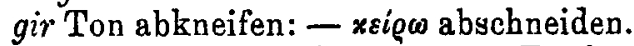
girin Töpfertonstück: - $x \in € \propto \mu \rho 5$ Töpferton.

3 gin hell glänzend = zagin (nà za.gin Lapislasuli, dieser heisst griechisch xvavós. $z a-\operatorname{seh} r=\zeta \alpha-$ sehr) gín $=$ xvavós blau.

$g i$ (ge?) Land: - roi $\alpha, \gamma \dot{\eta}$ Erde.

gu Stier, Rind: - s. gō Stier, Rind Boũs - lat. bos.

1 gur ein Verbum der Drehbewegung in vielen Nüancen: - rü @os Kreisbewegung.

5 gur Masstab, Mass: xógos Malter.

6 gur Eimer, Tonne (karu) - s. karaka Krug - xégvos Opferschüssel ksl. ¿̇ara Becher - and. hverr Topf.

8 gur dick: s. guru = lat. gravis schwer.

9 gur feind (nakru ša amāti!): — - s. garj anschreien, drohen - rogrós drohend.

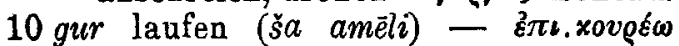
herbeieilen - lat. curro laufen.

11 gùr zerschneiden (zu gir): 一 xoũ œa Schur.

7 gur érnten, gurin Frucht: - lat. granum - ahd. chorn - ${ }_{n}$ Korn".

$\mathrm{Zu} \mathrm{k}$ : 1 ka $i z i$ brennen: - s. cona flammend

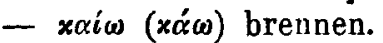

2 ka Frucht: - s. çāka Kraut.

ka Hase: - s. caca Hase = ags. hara, abd. haso Hase.

$1 \mathrm{kad}$ in al.kad ein netzartiges Gerät: s. kata Matte.

2 kad ein Fisch? nūn timri: «ïros Robbe, Delphin u. ä.

5 kin. Kin mahlen (hin und ber rollen des

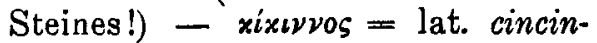
nus Locke.

keš Stirn: - s. keça Haupthaar - neupers. gēsō Locke.

$\mathrm{Zu} \mathrm{d:} 1 d a \bar{l}$ sich entfernen - $\tau \eta \lambda \dot{\vartheta} \vartheta \varepsilon \nu, \tau \eta \lambda_{0} \tilde{v}$ u. a. m. weit, fern.

3 dal glänzend hervortreten, glänzend: $\delta \alpha \lambda o ́ s$ Feuerbrand, $\delta \bar{\eta} \lambda o \varsigma$ offenbar u.ä.

dar einstürzen; einreissen: - s. dar zerbersten, zerreissen - $\delta \varepsilon ́ \omega(\delta \alpha \varrho \gamma \omega)$

- schinden.

(ki darra Erdspalt vgl. s. dāra Spalt, Riss.)

dam conjuñ̄: - $\delta \dot{\alpha} \mu \alpha \rho$ Gattin.
$2 d i$, deglänzen:-s.div strahlen, leuchten - dios glänzend - lat. divus.

5 dúbTafel (tuppu) 6 dúb Siegel eindrücken: - s. tup stossen - vú $\pi \tau \omega$ schlagen, stossen, stechen.

Zu t. 2 tab brennen: - s. tapas Wärme - lat. tepor Wärme.

1 til leben: - $\tau \dot{\varepsilon} \lambda \lambda \omega(\not ̈ \tau \varepsilon \iota \lambda \alpha)$ gedeihen.

2 til vollständig, zu Ende sein; beenden, vernichten; Ende; alt (sein): — s. taras ans Ziel kommen - $\tau \hat{\varepsilon} \lambda$ os Ende, Ziel, $\tau \varepsilon \lambda \dot{\varepsilon} \omega, \tau \varepsilon \lambda \varepsilon v \tau \alpha \dot{\omega}$ beenden u. ä.

3 til Webgeschrei. - $\tau i \lambda \lambda \omega$ prägn: unter Haarausraufen wehklagen.

Zu t. 3 tu Wind und

$t \bar{u}$, tūtū Beschwörung: - s. dhu anfächeln, anfachen - $\vartheta \dot{v} \omega$ opfern, $\vartheta v \sigma i \alpha$ Opfer $\vartheta v^{\prime} \omega$ stürmen, $\vartheta v^{\prime} \varepsilon \lambda \lambda \alpha$ Sturm.

3 tìr Hof (urspr. wohl Eintritt nach Delitzsch) auch Stall: - s. dur Tür

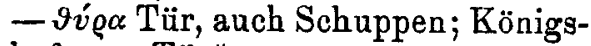
hof - Tü̈r"

Zu l u.r lub.a (wie zu lesen?) Fuchs: Die Lesung dürtte richtig sein, denn vgl. s. löpaças - $\alpha^{3} \lambda \omega \pi \eta \xi$ Fuchs. - lit. lápe, preuss. lape.

2 ra, 2rigehen : fübren 2 riwehen, stürmen: - s. ri los machen, laufen lassen u.ä. - @́śc tliessen.

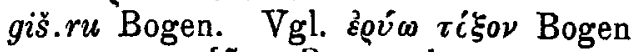

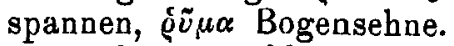

Zu m u. n 2 mar rings umschliessen, mer Gürtel,

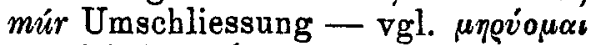
einwickeln, $\mu \dot{\eta} \varrho v \mu \alpha, \mu \dot{\eta} \varrho \iota \nu 90 \varsigma$ Band - lat. murus Mauer.

4 -mar anscheinend eine krankhafte Affektion - s. marana das Sterben +

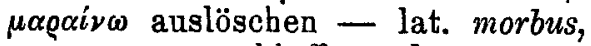
mors, marceo schlaff werden

mar.tu 1. wahrsch. Sturmwind 2. spez. Westwind - s. marut Wind. Gott des Windes vgl. 'Mar.tu als Bezeichnung Adads.

5 maš Gazellenbock: - s. mesha Widder.

2 na Mensch, Mann: - s. na Mann nar(?) nir Herr: s. nar Maun a’víe

1 nin Herrin, Fürstin; Priesterin; Schwester(?). - $\nu \varepsilon \tilde{a} \nu \iota \varsigma, \nu \tilde{\eta} \nu \iota \varsigma$ Jungfrau.

$\mathrm{Zu}$ h 4 hăr (so zu lesen?) ein Körperteil (haše Eingeweide?) - ved. hir $\bar{a}$ Darm -

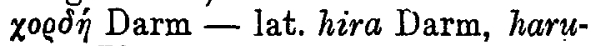
spex Eingeweideschauer.

har.sag Gebirge: - s. harsh starren, rauh sein - $\chi \varepsilon$ ćoos Festland (das Starre) - lat. horrere starren.

2 he schütten: - s. ju.hoti giesst $-\chi^{\hat{\epsilon} \omega}$, xów giessen; schütten. - got.giutan "giessen" 


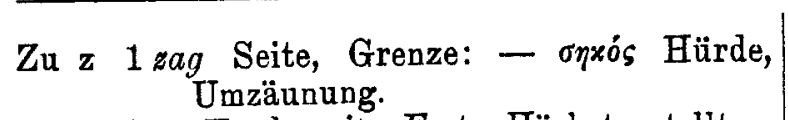

1 zag Vorderseite, Erster, Höchstgestellter: - ìyćsua anführen; áyı

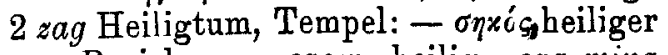
Bezirk. - sacer heilig, sag.mina heilig. Gras.

3 a. $z a g$ Macht, Kraft: - s. sahas Gewalt Vergewaltigung.

3 b. $z a g$ Entscheidung: - lat. sagio scharf wahrnehmen, sagax scharfsinnig

3 c. $z a g$ reden: - lat, secuta est = locuta est, sectus Rede.

$4 z a g$ Knie: - s. sagti Verbindung, sakthi Schenkel.

ziz Motte: - ớs Motte

$\mathrm{Zu}$ s: 1 sa Geflecht (als Urbedeutung) - $\alpha^{\prime} \omega$

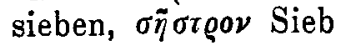

4 sa (urspr. viell. streichen) schlagen: $\psi \alpha \dot{\alpha} \omega$ streichen, reiben

1 sag Kopf, Anfang, Antlitz, Front u. ä. wohl identisch mit 1 zag s.d.

2 sir Licht: - s. sur leuchten, sur Sonne

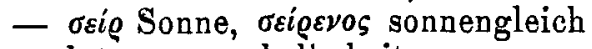
- lat. serenus hell, heiter.

4 sud Licht, nur vom Himmel gebraucht: - lat. sūdum klarer Himmelsraum.

$\mathrm{Zu}$ š: 2 šar Menge, Masse, Fülle u. ä.: - ahd. scara Menge, "Schar".

še.ir.zi Glanz: - and. skirr glänzend, hell - got. skeiras hell, deutlich.

1 šur regnen (lassen): - got. skura vindis Windschauer; and. skur Regen"schauer".

4 šür zornig - alts. scūr Kampf.

šurin tönerner Ofen: - lat. scoria Schlacke - mbd. scorstein, scorenš $=\sigma \pi \lambda$ ? stein "Schorn"stein.

2 šag Herz, Mitte; meton. Neigung Hunger - Wille - Begehr. Vgl. $\sigma \pi \lambda \alpha^{\prime} \gamma \chi^{\nu \alpha}$ die edleren Eingeweide, auch Herz; meton. Herz, Gemüt. $\sigma \pi \lambda$ anders als durch $\check{s}$ wiederzugeben, dürfte den Assyrern wohl unmöglich gewesen sein.

Der vorliegende Auszug aus dem viel umfangreicheren Material dürfte dartun, dass erhebliches indogermanisches Sprachgut in der sumerischen Sprache entbalten ist. Er dürfte vielleicht auch eine gewisse Stütze sein für folgende Behauptungen, die wegen Platzmangel nicht bewiesen werden können:

Dass die Konjugation des sumerischen Verbums merkliche Aehnlichkeiten mit der der griechischen Verben zeigt,

Dass die sumerischen Verbalpräformative den präpositionalen Präformativen des grie- chischen Verbums entsprechen, wie diese auch in Häufung beim gleichen Verbalstamm auftreten und sich lautlich und sinngemäss zum merklichen Teil damit identifizieren lassen,

Dass die sumerische Astronomie auf Grund der Wortvergleichung sich als grossenteils indogermanischen Ursprungs zu erweisen scheint.

Dafür, dass mir, als einem Neuling, unter den jetzigen schwierigen Verbältnissen hier der Raum zu einer kurzen Darlegung. gewäbrt wurde, fühle ich mich zu ganz besonderem Danke verpflichtet.

\section{Die Begräbnisstätte der heiligen Kühe von} Aphroditopolis (Ațîh).

\author{
Von W. Spiegelberg.
}

Wir wissen durch Strabo (XVII 35 [809]) 1 , dass in der Hauptstadt des 22. oberägyptischen Gaues Aphroditopolis, dem heutigen etwa $80 \mathrm{~km}$ südlich von Cairo auf dem östlichen Nilufer gelegenen Atfî̀n, eine weisse Kuh verehrt wurde. Strabo berichtet $z$ war genau genommen nur von der Verpflegung des lebenden beiligen Tieres, aber nach allem, was wir sonst über den ägyptischen Tierkultus wissen, dürfen wir ohne weiteres den Schluss ziehen, dass auch das tote Tier, also die einbalsamierte Leiche, hier die gleiche Verehrung genoss wie an anderen Orten, dass es also ähnlich wie z. B. bei den in Memphis verehrten Apisstieren auch in der Nähe von Aphroditopolis einen besonderen Grabbezirk für die heiligen Tiermumien gab. Dieses „Serapeum " von Aphroditopolis ist nun längst entdeckt worden, freilich ohne dass die glücklichen Finder es bemerkt haben.

Im Juni 1906 legte Ahmed Bey Kamal ${ }^{2}$ in Atfîh - nähere Angaben über die Oertlichkeit fehlen - ein bereits geplïindertes Grab der Ptolemäerzeit frei, das reich mit Darstellungen und Inschriften ausgestattet war und einen erbrochenen Sarg enthielt, über dessen Inhalt der Bericht nichts erwähnt. Dasselbe Grab wurde im Winter 1911-1912 von Ernest Mackay ${ }^{3}$ ohne Kenntnis des ersten Berichts aufs neue entdeckt und die Inschriften wurden vollständig und, soweit ich sehen kann, vortrefflich veröffentlicht, so dass sich jetzt auch die Frage nach dem Eigentümer des Grabes sicher beantworten lässt. Der erste Entdecker stand ihr nämlich ratlos ${ }^{4}$ gegenüber, während Mackay

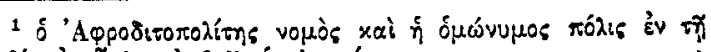

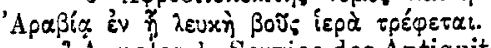

Annales du Service des Antiquités IX (1908) S. $113 \mathrm{ff}$.

3 Heliopolis, Kafr Ammar and Shurafa London 1915.

4 "Malgré la quantité de textes religieur qui on couvre" les parois, on n'y voit nulle part le nom da 